Kyushu J. Math.

Vol. 56, 2002, pp. 137-145

\title{
CURVES WITH ASYMPTOTIC GEODESICS
}

\author{
Kazuyuki ENOMOTO
}

(Received 28 August 2000, revised 15 February 2001 and accepted 26 February 2001)

Dedicated to Katsuhiro Shiohama on his sixtieth birthday

\section{Introduction}

Let $M$ be an $n$-dimensional simply connected complete Riemannian manifold of nonpositive sectional curvature. Let $\Sigma$ be a curve of class $C^{2}$ in $M$ and $x(s)$ be a parameterization of $\Sigma$ by arclength $s$. We assume that $x(s)$ is defined for all real numbers $s$. Let $T$ be a unit tangent vector of $\Sigma$ and let $D$ be the Levi-Civita connection of $M$. Then $k(s)=\left|D_{T} T\right|$ is called the geodesic curvature of $\Sigma$. Let $O$ be a fixed point in $M$ and let $r(s)=d(O, x(s))$, where $d$ is the distance in $M$.

$\Sigma$ is a geodesic if $k(s)=0$ for all $s$. In particular, in the $n$-dimensional Euclidean space $E^{n}$, if $k(s)=0$ for all $s, \Sigma$ is a straight line. However, the condition that $\lim _{s \rightarrow \infty} k(s)=0$ does not necessarily imply that $\Sigma$ looks 'straight' near infinity, as is seen from such an example as the curve defined by $r=\theta$ in the polar coordinate system $(r, \theta)$ in $E^{2}$.

In this paper, we give a sufficient condition for $\Sigma$ to have an asymptotic geodesic (asymptotic line) on its end. Here an asymptotic geodesic means a geodesic $\Gamma: y(t)$ which has the property that the function $h(s):=\inf _{t} d(x(s), y(t))$ tends to zero as $s \rightarrow+\infty$ or $s \rightarrow-\infty$. We prove that if $\Sigma$ is in $E^{n}$ and satisfies $\int_{-\infty}^{\infty} k(s) r(s) d s<$ $\infty$, then $\Sigma$ has an asymptotic line on each end (Theorem 3.1). We also show that if $\Sigma$ is in the $n$-dimensional hyperbolic space $H^{n}$ and satisfies $\int_{-\infty}^{\infty} k(s) d s<\infty$, then $\Sigma$ has an asymptotic geodesic on each end (Theorem 2.1). Theorem 3.1 is a generalization of a result in [E] for curves in $E^{2}$.

If a curve $\Sigma$ in $E^{n}$ satisfies the condition that $\lim _{s \rightarrow \infty} r(s)=\infty$ (i.e. properly immersed) and $k(s) r(s)^{2+\varepsilon}$ is uniformly bounded with some positive constant $\varepsilon$, then $\int_{-\infty}^{\infty} k(s) r(s) d s<\infty$ holds and $\Sigma$ has an asymptotic line on each end (Remark 3.2). In general, of course, $k(s)$ may not converge to zero as $s \rightarrow \pm \infty$ under the condition that $\int_{-\infty}^{\infty} k(s) r(s) d s<\infty$.

Although our main results are for curves in $E^{n}$ or $H^{n}$, we study some asymptotic behaviors of curves with $\int_{-\infty}^{\infty} k(s) d s<\infty$ in a Riemannian manifold of non-positive sectional curvature (Section 1), which are used to prove our main theorems. 


\section{Curves in manifolds of non-positive curvature}

In this section, we prove the following proposition.

PROPOSITION 1.1. Let $\Sigma: x(s)(-\infty<s<\infty)$ be a curve in a simply connected complete Riemannian manifold $M$ of non-positive sectional curvature. Let $O$ be a fixed point in $M$ and let $r(s)=d(O, x(s))$. If $\int_{-\infty}^{\infty} k(s) d s<\infty$, then

$$
\lim _{s \rightarrow \pm \infty}\left|\frac{d r}{d s}\right|=1 .
$$

In particular, $\lim _{s \rightarrow \pm \infty} r(s)=\infty$, i.e. $\Sigma$ is properly immersed.

Proof. Since $\int_{-\infty}^{\infty} k(s) d s<\infty$, for any positive constant $\varepsilon<\pi / 2$, there exists $s_{0}$ such that

$$
\int_{s_{0}}^{s_{1}} k(s) d s<\varepsilon .
$$

holds for any $s_{1}>s_{0}$. Let $\Sigma_{1}$ be a piecewise $C^{2}$ closed curve consisting of $\left\{x(s): s_{0} \leq s \leq s_{1}\right\}$ and the geodesic segment $\gamma$ joining $x\left(s_{0}\right)$ and $x\left(s_{1}\right)$. Let $\varphi_{0}$ be the angle between $T\left(s_{0}\right)$ and $\gamma$ and let $\varphi_{1}$ be the angle between $T\left(s_{1}\right)$ and $\gamma$. The total absolute curvature of $\Sigma_{1}$ is given by

$$
\int_{s_{0}}^{s_{1}} k(s) d s+\left(\pi-\varphi_{0}\right)+\left(\pi-\varphi_{1}\right) .
$$

Since, by a theorem of Szentze $[\mathbf{S}]$, the total absolute curvature of any piecewise $C^{2}$ closed curve in a Riemannian manifold of non-positive sectional curvature is not less than $2 \pi$, we have

$$
\varphi_{0}+\varphi_{1} \leq \int_{s_{0}}^{s_{1}} k(s) d s<\varepsilon .
$$

Hence we have

$$
0 \leq \varphi_{1}<\varepsilon<\frac{\pi}{2}
$$

For $x$ in $M$ let $r_{0}(x)=d\left(x\left(s_{0}\right), x\right)$. Regarding $r_{0}$ as a function on $M$, we denote the gradient vector field of $r_{0}$ by $\nabla r_{0}$. Then we have

$$
\begin{aligned}
\left.\frac{d}{d s} r_{0}(x(s))\right|_{s=s_{1}} & =\left\langle\nabla r_{0}\left(x\left(s_{1}\right)\right), T\left(s_{1}\right)\right\rangle \\
& =\cos \varphi_{1} \\
& >\cos \varepsilon .
\end{aligned}
$$


Since $\varepsilon$ is any positive constant, this shows that

$$
\lim _{s \rightarrow \infty} \frac{d}{d s} r_{0}(x(s))=1 .
$$

Since

$$
r_{0}(x(s))-r\left(s_{0}\right) \leq r(s) \leq r_{0}(x(s))+r\left(s_{0}\right),
$$

(1.1) implies that

$$
\lim _{s \rightarrow \infty} \frac{d r}{d s}=1
$$

Similarly, we have

$$
\lim _{s \rightarrow-\infty} \frac{d r}{d s}=1 .
$$

and the proof for Proposition 1.1 is complete.

To state the following lemma, let $\gamma_{s}$ be the oriented geodesic of $M$ from $O$ to $x(s)$ and let $X(s)$ be the unit tangent vector of $\gamma_{s}$ at $O . X(s)$ is regarded as a curve in the unit $(n-1)$-sphere consisting of all unit tangent vectors of $M$ at $O$.

LEMMA 1.2. If $\int_{-\infty}^{\infty} k(s) d s<\infty$, then $X(s)$ converges as $s \rightarrow \pm \infty$.

Proof. For $x$ in $M$ let $r(x)=d(O, x)$. Regarding $r$ as a function on $M$, the gradient vector $\nabla r$ is the unit tangent vector of $\gamma_{s}$. Let $\theta(s)$ be the angle between $\gamma_{s}$ and $\Sigma$ at $x(s)$. Then we have

$$
\begin{aligned}
\frac{d}{d s} r(x(s)) & =\langle\nabla r(x(s)), T(s)\rangle \\
& =\cos \theta(s) .
\end{aligned}
$$

It follows from (1.2) and Proposition 1.1 that

$$
\lim _{s \rightarrow \infty} \theta(s)=0
$$

Let $\varepsilon$ be any positive constant. Then there exists $s_{0}$ such that for any $s_{1}>s_{0}$

$$
\int_{s_{0}}^{s_{1}} k(s) d s<\frac{\varepsilon}{3}, \quad \theta\left(s_{0}\right)<\frac{\varepsilon}{3}, \quad \theta\left(s_{1}\right)<\frac{\varepsilon}{3} .
$$

Since, by Szenthe's theorem [S], the total absolute curvature of the piecewise $C^{2}$ closed curve consisting of $\gamma_{s_{0}}, \gamma_{s_{1}}$ and $\left\{x(s): s_{0} \leq s \leq s_{1}\right\}$ is not less than $2 \pi$, we have

$$
\int_{s_{0}}^{s_{1}} k(s) d s+\theta\left(s_{0}\right)+\pi-\theta\left(s_{1}\right)+\pi-d_{S}\left(X\left(s_{0}\right), X\left(s_{1}\right)\right) \geq 2 \pi,
$$


where $d_{S}$ is the distance in the unit $(n-1)$-sphere of all unit tangent vectors of $M$ at $O$. (1.4) and (1.5) give

$$
d_{S}\left(X\left(s_{0}\right), X\left(s_{1}\right)\right) \leq \int_{s_{0}}^{s_{1}} k(s) d s+\theta\left(s_{0}\right)-\theta\left(s_{1}\right)<\varepsilon .
$$

Hence $X(s)$ converges to a unit tangent vector $X_{\infty}$ of $M$ at $O$ as $s \rightarrow \infty$. The case when $s \rightarrow-\infty$ is similar.

\section{Curves in hyperbolic space}

In this section, we prove the following theorem.

THEOREM 2.1. Let $\Sigma: x(s)$ be a curve of class $C^{2}$ in $H^{n}$ which is parameterized by arclength $s$ for $-\infty<s<\infty$. If $\int_{-\infty}^{\infty} k(s) d s<\infty$, then $\Sigma$ has an asymptotic geodesic on each end.

Proof. Let $\Gamma$ be the geodesic of $H^{n}$ through $O$ which is tangent to $X_{\infty}$ at $O$, where $X_{\infty}$ is the unit tangent vector of $H^{n}$ at $O$ given in Lemma 1.2. Let $y(s)$ be the point on $\Gamma$ such that $d(O, y(s))=r(s) . x(s)$ and $y(s)$ are joined by a curve $\left\{\exp _{O}(r(s) X(t)): s \leq t<\infty\right\}$, whose length shall be denoted by $\ell(s)$. Let $\tilde{\ell}(s)$ be the length of the curve $\{X(t): s \leq t<\infty\}$ which lies in the unit sphere consisting of all unit tangent vectors of $H^{n}$ at $O$. Then we have

$$
\ell(s)=\sinh (r(s)) \tilde{\ell}(s) .
$$

Let $d \tilde{s}$ be the line element of $X(t)$. The Riemannian metric of $H^{n}$ is written as

$$
d s^{2}=d r^{2}+\sinh ^{2} r d \tilde{s}^{2} .
$$

It follows from (1.2) and (2.2) that

$$
\sin \theta(s) d s=\sinh (r(s)) d \tilde{s} .
$$

Since $\lim _{s \rightarrow \infty} r(s)=\infty$ by Proposition 1.1, we use l'Hospital's rule together with Proposition 1.1, (1.2), (2.1) and (2.3) to derive

$$
\begin{aligned}
\lim _{s \rightarrow \infty} \ell(s) & =\lim _{s \rightarrow \infty} \frac{\tilde{\ell}(s)}{1 / \sinh (r(s))} \\
& =\lim _{s \rightarrow \infty} \frac{d \tilde{\ell} / d s}{-(d r / d s) \cosh (r(s)) / \sinh ^{2}(r(s))} \\
& =\lim _{s \rightarrow \infty} \frac{-(d \tilde{s} / d s) \sinh (r(s))}{-(d r / d s) \cosh (r(s)) / \sinh (r(s))}
\end{aligned}
$$




$$
\begin{aligned}
& =\lim _{s \rightarrow \infty} \frac{d \tilde{s}}{d s} \sinh (r(s)) \\
& =\lim _{s \rightarrow \infty} \sin \theta(s) \\
& =0 .
\end{aligned}
$$

Since $d(x(s), \Gamma) \leq d(x(s), y(s)) \leq \ell(s),(2.4)$ implies

$$
\lim _{s \rightarrow \infty} d(x(s), \Gamma)=0 .
$$

Hence $\Gamma$ is an asymptotic geodesic of $\Sigma$ for $s \rightarrow \infty$. By a similar argument, one can show that $\Sigma$ has an asymptotic geodesic for $s \rightarrow-\infty$.

Remark 2.1. Asymptotic geodesic on each end is not unique. In fact, in $H^{n}$ there are infinitely many geodesics which are asymptotic to one geodesic.

Remark 2.2. It is natural to ask if the above theorem holds for curves in $M$ whose sectional curvature $K_{M}$ satisfies $-b^{2} \leq K_{M} \leq-a^{2}<0$ with some constants $a$ and $b(0<a<b)$. We do not know the answer, but it seems that the proof given above does not work in this situation, as we explain below. If $-b^{2} \leq K_{M} \leq-a^{2}<0$, then we have

$$
\sinh (\operatorname{ar}(s)) \tilde{\ell}(s) \leq \ell(s) \leq \sinh (\operatorname{br}(s)) \tilde{\ell}(s)
$$

instead of (2.1), and also

$$
\sinh (\operatorname{ar}(s)) d \tilde{s} \leq \sin \theta(s) d s \leq \sinh (\operatorname{br}(s)) d \tilde{s}
$$

instead of (2.3). As a result, we have

$$
\begin{aligned}
\lim _{s \rightarrow \infty} \ell(s) & \leq \lim _{s \rightarrow \infty} \frac{\tilde{\ell}(s)}{1 / \sinh (r(s))} \\
& =\lim _{s \rightarrow \infty} \frac{d \tilde{s}}{d s} \sinh (b r(s)) \\
& \leq \lim _{s \rightarrow \infty} \frac{\sinh (\operatorname{br}(s))}{\sinh (\operatorname{ar}(s))} \sin \theta(s)
\end{aligned}
$$

instead of (2.4). If $0<a<b$, there is no guarantee that

$$
\lim _{s \rightarrow \infty} \frac{\sinh (b r(s))}{\sinh (\operatorname{ar}(s))} \sin \theta(s)=0
$$

and the existence of asymptotic geodesics cannot be derived from this argument. 


\section{Curves in Euclidean space}

For curves in $E^{n}$, the condition $\int_{-\infty}^{\infty} k(s) d s<\infty$ is not sufficient to have asymptotic lines, as we see from such an example as the graph of $y=x^{2}$ in $E^{2}$. In the following theorem, we will give a sufficient condition for $\Sigma$ in $E^{n}$ to have asymptotic lines. For curves in $E^{2}$ this theorem is proved in $[\mathbf{E}]$.

THEOREM 3.1. Let $\Sigma: x(s)$ be a curve in $E^{n}$ which is parameterized by arclength $s$ for $-\infty<s<\infty$. If $\int_{-\infty}^{\infty} k(s) r(s) d s<\infty$, then $\Sigma$ has an asymptotic line on each end.

Proof. Since $\Sigma$ is a curve in the Euclidean space, we have

$$
\frac{d x}{d s}=T(s), \quad \frac{d^{2} x}{d s^{2}}=\frac{d T}{d s}=k(s) N(s),
$$

where $N(s)$ is a unit normal vector of $\Sigma$. Let $x^{\perp}(s)=x(s)-\langle x(s), T(s)\rangle T(s)$. For any $\varepsilon>0$, there exists $s_{0}>0$ such that

$$
\int_{s_{1}}^{s_{2}} k(s) r(s) d s<\varepsilon
$$

holds for any $s_{1}, s_{2} \geq s_{0}$. Since

$$
\begin{aligned}
\left|\frac{d x^{\perp}}{d s}\right|^{2} & =\left|T-\langle T, T\rangle T-\left\langle x, \frac{d T}{d s}\right\rangle T-\langle x, T\rangle \frac{d T}{d s}\right|^{2} \\
& =|-\langle x, k N\rangle T-\langle x, T\rangle k N|^{2} \\
& =k^{2}\left(\langle x, N\rangle^{2}+\langle x, T\rangle^{2}\right) \\
& \leq k(s)^{2}|x(s)|^{2} \\
& =k(s)^{2} r(s)^{2},
\end{aligned}
$$

we have

$$
\begin{aligned}
\left|x^{\perp}\left(s_{2}\right)-x^{\perp}\left(s_{1}\right)\right| & =\left|\int_{s_{1}}^{s_{2}} \frac{d x^{\perp}}{d s} d s\right| \\
& \leq \int_{s_{1}}^{s_{2}}\left|\frac{d x^{\perp}}{d s}\right| d s \\
& \leq \int_{s_{1}}^{s_{2}} k(s) r(s) d s \\
& <\varepsilon .
\end{aligned}
$$


This shows that $\lim _{s \rightarrow \infty} x^{\perp}(s)$ exists. Set $x_{\infty}^{\perp}=\lim _{s \rightarrow \infty} x^{\perp}(s)$.

We can also show that $\lim _{s \rightarrow \infty} T(s)$ exists. In fact, if $s_{0}$ is sufficiently large, we have $r(s)>1$ for all $s \geq s_{0}$ and

$$
\begin{aligned}
\left|T\left(s_{2}\right)-T\left(s_{1}\right)\right| & =\left|\int_{s_{1}}^{s_{2}} \frac{d T}{d s} d s\right| \\
& \leq \int_{s_{1}}^{s_{2}}\left|\frac{d T}{d s}\right| d s \\
& =\int_{s_{1}}^{s_{2}} k(s) d s \\
& <\int_{s_{1}}^{s_{2}} k(s) r(s) d s \\
& <\varepsilon .
\end{aligned}
$$

Set $T_{\infty}=\lim _{s \rightarrow \infty} T(s)$.

We define a straight line $\Gamma: y(t)$ by $y(t)=x_{\infty}^{\perp}+t T_{\infty}$. Let $\bar{x}(s)$ be a point on $\Gamma$ which is defined by

$$
\bar{x}(s)=x_{\infty}^{\perp}+\left\langle x(s), T_{\infty}\right\rangle T_{\infty}
$$

$\Gamma$ becomes an asymptotic line of $\Sigma$ if and only if

$$
\lim _{s \rightarrow \infty}|x(s)-\bar{x}(s)|=0 .
$$

Since $x(s)=x^{\perp}(s)+\langle x(s), T(s)\rangle T(s)$, we have

$$
\begin{aligned}
|x(s)-\bar{x}(s)| & =\left|\left(x^{\perp}(s)-x_{\infty}^{\perp}\right)+\langle x(s), T(s)\rangle T(s)-\left\langle x(s), T_{\infty}\right\rangle T_{\infty}\right| \\
& =\left|\left(x^{\perp}(s)-x_{\infty}^{\perp}\right)+\langle x(s), T(s)\rangle\left(T(s)-T_{\infty}\right)+\left\langle x(s), T(s)-T_{\infty}\right\rangle T_{\infty}\right| \\
& \leq\left|x^{\perp}(s)-x_{\infty}^{\perp}\right|+|\langle x(s), T(s)\rangle|\left|T(s)-T_{\infty}\right|+\left|\left\langle x(s), T(s)-T_{\infty}\right\rangle\right| \\
& \leq\left|x^{\perp}(s)-x_{\infty}^{\perp}\right|+2 r(s)\left|T(s)-T_{\infty}\right| .
\end{aligned}
$$

Since $\int_{0}^{\infty} k(s) r(s) d s$ converges, for any $\varepsilon>0$, there exists $s_{0}$ such that

$$
\int_{s_{0}}^{\infty} k(s) r(s) d s<\varepsilon
$$


By Proposition 1.1, we may assume that $d r / d s>0$ for all $s \geq s_{0}$. This implies that, if $t \geq s \geq s_{0}$, then $r(t)>r(s)$. Now we have, for any $s \geq s_{0}$,

$$
\begin{aligned}
r(s)\left|T(s)-T_{\infty}\right| & =r(s)\left|\int_{s}^{\infty} \frac{d T}{d t} d t\right| \\
& \leq r(s) \int_{s}^{\infty}\left|\frac{d T}{d t}\right| d t \\
& =r(s) \int_{s}^{\infty} k(t) d t \\
& \leq \int_{s}^{\infty} k(t) r(t) d t \\
& <\varepsilon .
\end{aligned}
$$

Hence

$$
\lim _{s \rightarrow \infty}|x(s)-\bar{x}(s)| \leq \lim _{s \rightarrow \infty}\left(\left|x^{\perp}(s)-x_{\infty}^{\perp}\right|+2 r(s)\left|T(s)-T_{\infty}\right|\right)=0 .
$$

This shows that $\Gamma$ is an asymptotic line of $\Sigma$ for $s \rightarrow \infty$. Similarly, $\Sigma$ has an asymptotic line for $s \rightarrow-\infty$.

Remark 3.1. As we see from the proof, $\lim _{s \rightarrow \infty} T(s)$ exists under the condition that $\int_{-\infty}^{\infty} k(s) d s<\infty$.

Remark 3.2. If $\Sigma$ in $E^{n}$ satisfies the condition that $\lim _{s \rightarrow \infty} r(s)=\infty$ (i.e. properly immersed) and $k(s) r(s)^{2+\varepsilon} \leq A$ for all $s$ with some positive constants $\varepsilon$ and $A$, then by a result in $[\mathbf{K}]$ there exist positive constants $B$ and $s_{0}$ such that $|d r / d s| \geq B$ for all $s$ with $|s| \geq s_{0}$. Thus we have

$$
\begin{aligned}
\int_{-\infty}^{\infty} k(s) r(s) d s & \leq \int_{-\infty}^{\infty} \frac{A}{r(s)^{1+\varepsilon}} d s \\
& \leq \int_{-s_{0}}^{s_{0}} \frac{A}{r(s)^{1+\varepsilon}} d s+\int_{r\left(-s_{0}\right)}^{\infty} \frac{A}{B r^{1+\varepsilon}} d r+\int_{r\left(s_{0}\right)}^{\infty} \frac{A}{B r^{1+\varepsilon}} d r \\
& <\infty .
\end{aligned}
$$

Hence $\Sigma$ has an asymptotic line on each end. This shows how fast the geodesic curvature should decay to have asymptotic lines, when one controls the geodesic curvature pointwise. However, our theorem is more general because we do not need pointwise estimates for the geodesic curvature. Our theorems do not need the assumption that $\Sigma$ is properly immersed, either. 
Acknowledgement. The author expresses his thanks to the referee for his helpful comments.

\section{REFERENCES}

[E] K. Enomoto. Plane curves with asymptotic lines. Kyushu J. Math. 49 (1995), 317-319.

[K] A. Kasue. Gap theorems for minimal submanifolds of Euclidean space. J. Math. Soc. Japan 38 (1986), 473-492.

[S] J. Szenthe. On the total curvature of closed curves in Riemannian manifolds. Publ. Math. Debrecen 15 (1968), 99-105.

Kazuyuki Enomoto

Faculty of Industrial Science and Technology

Science University of Tokyo

Oshamambe, Hokkaido 049-3514 Japan

(E-mail: enomoto@rs.kagu.sut.ac.jp) 